\title{
Olfactory Abnormalities and COVID; An Analysis of Hundred Cases
}

\section{Minutha $\mathbf{R}^{1 *}$ and Sriram Nathan ${ }^{2}$}

${ }^{1}$ Associate Professor, Department of ENT at Vydehi Medical College and Research Hospital, Bangalore, India

${ }^{2}$ Head and Neck and ENT Surgeon at Manipal Hospital, Bengaluru

*Corresponding Author: Minutha R, Associate Professor, Department of ENT at Vydehi Medical College and Research Hospital, Bangalore, India.
Received: July 09, 2021

Published: August 05, 2021

(C) All rights are reserved by Minutha $\mathbf{R}$ and Sriram Nathan.

\begin{abstract}
Introduction: With the recent pandemic of COVID Severe acute respiratory syndrome or SARS on the rise especially in the city, there has been an increase in the number of patients especially in the ENT out patient department with diagnosis of COVID. A common symptom of COVID and now a major symptom for diagnosis was the presence of loss of smell and taste to varying degree.

We examined those patients with proven diagnosis of COVID and analysed the symptoms of Anosmia or Hyposmia in them. This is a short term study of three months where we analysed the patients encountered by the author in the time period.

Over one hundred patients were encountered during this period and their assessment in respect to COVID was done and results presented. Some patients were seen in the out patient department, some in the operation theatre and during assessment as part of COVID triage.
\end{abstract}

Materials and Methods: Patients were screened for common symptoms of COVID with special emphasis on olfactory anomalies and followed up.

Observation: Olfactory symptoms were seen in a high number of patients (over 40 percent); this could be partially since the ENT practice will encounter these symptoms first.

Conclusion: The study makes the conclusion that this is an established fact that Olfactory anomalies especially sudden Anosmia is an important indicator of COVID infection. Further evaluation is needed for the pathophysiology and treatment protocols and this will be more clear in the coming months.

Keywords: COVID; Hyposmia; Anosmia

\section{Abbreviations}

COVID: Corona Virus Disease; SARS: Severe Acute Respiratory Syndrome; ENT: Ear Nose and Throat; RTPCR: Reverse Transcription Polymerase Chain Reaction

\section{Introduction}

The interesting and peculiar thing about the olfactory system is that the nerve endings are almost afferent nerve fibres sending its signal to a sensory neuron at the olfactory area of the nose and thus is very prone and sensitive to insult. Even a common cold is associated with smell abnormalities which can be temporary or permanent. In these cases though the smell abnormalities have been known to be conductive in nature and temporary. Since the nerve endings are in direct communication with the brain it has also been postulated as a conduit for diseases such as Alzheimer's and other viral infections. 
A trend seen in the recent pandemic of COVID 19; it was observed that one of the presenting complaints was olfactory abnormalities which was more prominent than what experienced in common flu and more acute in nature. Very soon it was also added as a major diagnostic criteria by the WHO [1-5].

We also experienced similar situation while dealing with patients with diagnosis of COVID. Many time the only symptom along with a positive RTPCR test for COVID were the olfactory abnormalities and this was associated with altered taste also in many cases since both smell and taste go together.

We thus analysed the olfactory abnormalities and taste alterations along with other symptoms in hundred patients diagnosed with COVID.

\section{Materials and Methods}

Patients who presented to the out patient department with different symptoms ranging from olfactory abnormalities and taste disturbances or loss, fever, body pain, and breathlessness were subjected to the COVID RTPCR testing. There were many who had no major symptoms who got the test done as per preoperative assessment and for being the primary or secondary contact. Those who tested positive were evaluated for the symptoms and recovery with special emphasis on the olfactory and taste anomalies. They were then questioned about the treatment received and also followed up for the recovery which was defined by a negative COVID RTPCR testing after an asymptomatic period of one week.

\section{Inclusion criteria}

Patients who were included in this study were:

- Adult patients over the age of 12 years with olfactory abnormalities.

- Patients who were tested positive for COVID with the RTPCR method.

- Patients with symptoms such as Anosmia, hyposmia and other symptoms not limited to the upper respiratory tract and/or ENT.

\section{Exclusion criteria}

- Children and infants who were tested positive.

- Patients with only antigen tested positivity since this was not a confirmatory test.
- Patients with pre existing olfactory anomalies and disturbances due to causes such as polyps and causes of permanent olfactory loss that is patients with chronic history of olfactory abnormalities.

The selected patients who met the inclusion criteria as enumerated above were followed up with a set of questions to assess their acute olfactory abnormalities secondary to COVID.

\section{Observations and Results}

We had 100 patients who were randomly included in the study. There were 47 males and 53 females in the study (Figure 1).

\section{Figure 1}

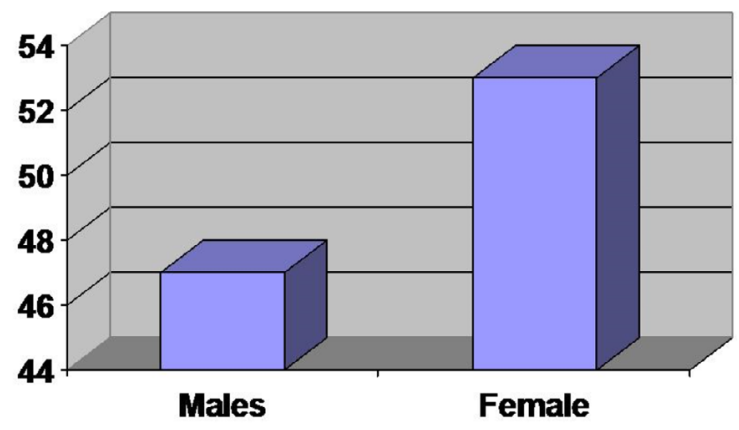

Figure 1

There were guidelines issued by the state and central Governments which was updated and modified regularly. The broad guidelines as summarized in the figure below. Most patients were managed in home management and isolation. Some with mild symptoms especially those who did not have situation ideal for home isolation were shifted to the state approved COVID care centre where they were monitored and treated according to the symptoms. Some were shifted to the hospital where the indication was risk factors and more symptoms for proper monitoring and treatment. The ones who were having more symptoms and having more risk factors were shifted to either High dependent unit or the ICU (Figure 2).

The age group ranged from the youngest patient of age 22 years while the oldest patient was 72 years old. The major age group was 35 - 45 years of age (Figure 3 ). 
Triage Protocol - Matrix (Updated - 22/07/20)

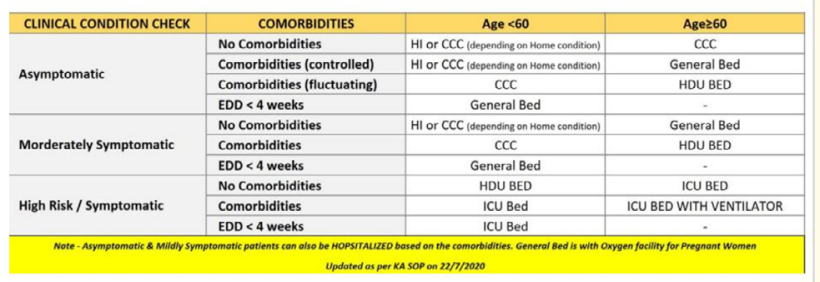

Figure 2

Figure 3- Age distribution

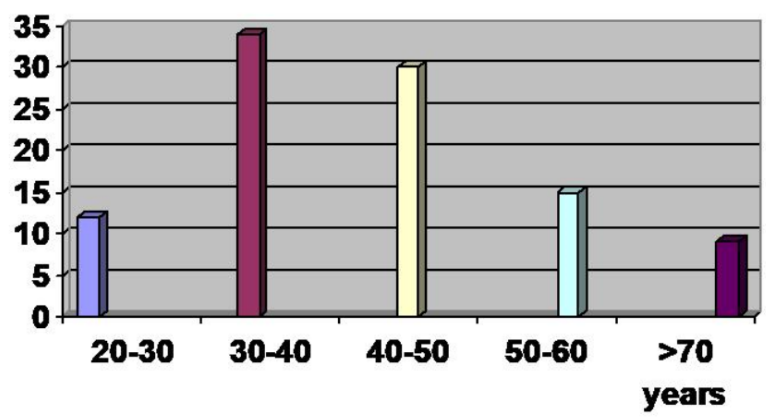

Figure 3

The most common symptom was generalised body pain and weakness which was seen in almost all the patients which was followed by fever, olfactory abnormalities, breathlessness, loose stools and cough (Figure 4).

Most patients especially those who were mildly symptomatic or asymptomatic were advised home isolation and home management, some were managed in the COVID CARE CENTRE while some rarely required hospitalisation, five patients were managed in ICU and two of them succumbed to the infection after a drastic course ending in multi organ failure and finally death (Figure 5).

The most common olfactory abnormality was Anosmia while others were Hyposmia, taste abnormalities were also seen. A total
Figure 4

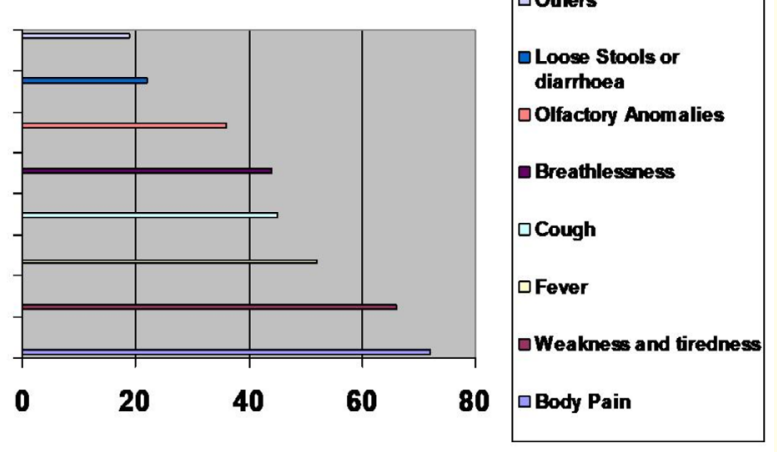

Figure 4
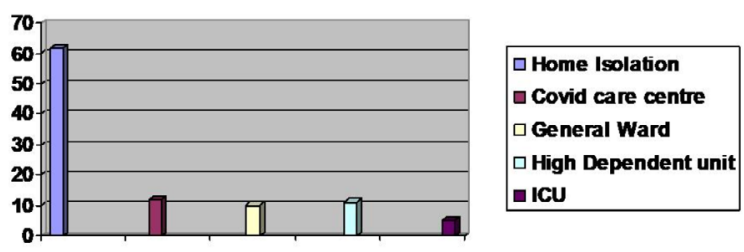

Figure 5 of 36 (14 Hyposmia and 22 Anosmia) percent of patients had some form of olfactory anomalies or the other. It was seen that almost 90 percentage of patients with Anosmia also mentioned taste alteration. While around 50 percentage of patients with Hyposmia had taste alteration. It is understood that severe smell abnormalities are associated with more prominent taste anomalies.

A Chi square analysis of the prevalence of olfactory anomalies in our study revealed $\mathrm{p}=0.00336457$ (Significant at $\mathrm{p}<.05$ ) which was clinically significant thus indicating that olfactory anomalies are a significant symptom in COVID (Figure 6).

The percentage of patients with olfactory anomalies were more in females ( 26 females and 10 males). 


\section{Figure 6}

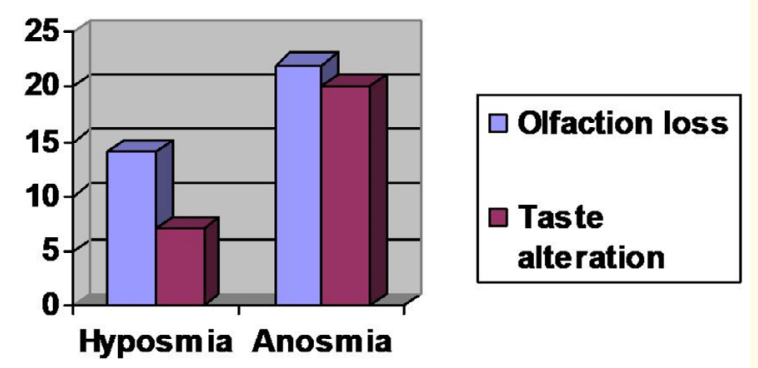

Figure 6

The chi-square statistic is 8.3436 . The $p$-value is .00387 . Significant at $\mathrm{p}<.05$.

The chi-square statistic with Yates correction is 7.1815. The pvalue is .007366 . Significant at $p<.05$. The statistical analysis thus reveals that the difference is not significant.

Two cases of the 36 were having septal abscess and Hyposmia, they were subsequently treated for the same and there was spontaneous recovery of the smell abnormality. The rest of the cases had no local abnormality or reason for a conductive loss of smell and were thus classified as sensory.

Since the author sees majority of patients with any olfactory or taste related problems it is understood that there is a speciality bias in the selection of the cases.

Majority recovered from the olfactory abnormalities while just two had permanent olfactory loss. One patient had a partial recovery after the first week only to have a permanent loss after one week. She still does not have the sense of smell. She was retested and it was revealed that she developed re infection. The follow up after three weeks revealed negativity of test again but the Olfactory loss continues.

There were two who succumbed to COVID, both did not have any olfactory loss or complaints. Both the patients were diabetic, Hypertensive and had poor cardiac status. They were treated in sequence in high dependent unit and then ICU where they were in- cubated and subsequently done tracheotomy for prolonged ventilation by the senior author. They had a prolonged course of around 4 weeks in the ICU after which they developed multi organ failure and death.

To assess the prognostic nature of Olfactory loss a Chi square analysis was done which revealed that though all the patients with olfactory anomalies recovered from the infection the association may not be significant ( $p=0.924213$ ).

\section{Discussion}

The amelioration of the current COVID pandemic relies on swift and efficient case finding as well as stringent social distancing measures. Current advice suggests that fever or new onset dry cough are the commonest presenting complaints.

Simon., et al. presented a case report and case series as well as other evidence that there is an important fourth presenting syndrome, namely isolated sudden onset Anosmia, which should be considered highly suspicious for SARS-CoV-2.

They examined a patient presenting with ISOA who went on to test positive for infection with COVID-19 and did not develop any further symptoms as well as a case series of similar patients although limited by the lack of reliable testing at the moment. In conclusion they insisted the existence of a fourth common syndrome of COVID-19 infection: isolated sudden onset Anosmia and urge the international community to consider this presentation in current management advice.

In our study although many were asymptomatic, body pain and weakness were the most common symptoms. The others symptoms include fever and cough. Our study though was more focussed on the olfactory anomalies so other symptoms were not assessed much [6].

Albert Y., et al. have in their article done an extensive assessment of the mechanism of olfactory anomalies in COVID. They observed that the global pandemic of corona virus disease 2019 (COVID-19) caused by severe acute respiratory syndrome corona virus 2 (SARS-CoV-2) remains a challenge for prevention due to asymptomatic or paucisymptomatic patients. Anecdotal and preliminary evidence from multiple institutions shows that these patients present with a sudden onset of Anosmia without rhinitis. They aimed 
to review the pathophysiology of Anosmia related to viral upper respiratory infections and the prognostic implications.

Preliminary evidence reveals that sudden Anosmia might be the sole presenting symptom of COVID-19 patients (Gane., et al. 2020; Hopkins., et al. 2020). In 214 hospitalized COVID-19 patients in Wuhan, 5.1\% and 5.6\% of patients presented with Hyposmia and hypogeusia, respectively (Mao., et al. 2020). An anecdotal survey of patients in South Korea revealed that about 30\% had Anosmia as their major presenting symptom of COVID-19 (ENT UK 2020). Furthermore, these patients presented with Anosmia and ageusia associated with fever $\left(>37.5^{\circ} \mathrm{C}\right)$ without nasal obstruction or rhinitis.

After these initial reports, the American Academy of Otolaryngology-Head and Neck Surgery, ENT UK and the British Rhinological Society independently published guidelines that include Anosmia, Hyposmia and dysgeusia in assessing patients suspected to have COVID-19 (ENT UK 2020). More recent olfactory surveys on COVID-19 patients showed olfactory dysfunction in 20 - 85\% of patients (cross referenced in their article). Magnetic resonance imaging of a patient with SARS-CoV-2-related isolated sudden Anosmia revealed normal olfactory bulb volume and signal intensity. About $72.6 \%$ of these patients recovered olfactory function within the first 8 days, which suggests that the majority of Anosmia is temporary in nature. A similar finding was confirmed using the University of Pennsylvania Smell Identification Test. It is possible that the apparently increasing incidence of olfactory dysfunction is due to greater awareness and more careful assessment of the symptom.

With few studies published yet, they could only speculate on the mechanism of Anosmia symptoms in SARS-CoV-2 patients. We can glean understanding from other respiratory viral infections, including other corona viruses in particular. Anosmia can be broadly categorized into conductive or sensorineural olfactory loss. Conductive loss occurs due to impaired nasal airflow and is reversible when the obstruction clears; sensorineural loss implies dysfunction of the $\mathrm{OE}$ and can be permanent or have a longer time course to functional recovery.

They finally concluded that Current evidence suggested that SARS-CoV-2-related Anosmia may be a new viral syndrome specific to COVID-19 and can be mediated by intranasal inoculation of SARS-CoV-2 into the olfactory neural circuitry. The clinical course of neuroinvasion of SARS-CoV-2 is yet unclear; however, an extend- ed follow-up of these patients to assess for neurological sequelae, including encephalitis, cerebrovascular accidents, and long-term neurodegenerative risk may be indicated.

In our study also smell anomalies were many times associated with taste anomalies as many times they go together. Further analysis is required on the pathophysiology of the reason of olfactory loss and this will be helpful in the future [7].

Katherine Lisa., et al. observed that as of May 1, 2020, more than 3000000 people worldwide have been infected with the novel corona virus, severe acute respiratory syndrome corona virus 2 (SARS-CoV-2). The CDC has highlighted key symptoms that may suggest corona virus disease 2019 (COVID-19), including cough, shortness of breath or difficulty breathing, fever, chills, muscle pain, sore throat, and new loss of smell or taste.

The inclusion of loss of smell or taste among these symptoms follows the emergence of evidence that suggests that COVID-19 frequently impairs the sense of smell. For example, they observed that in a study from Iran, 59 of 60 patients hospitalized with COVID-19 were found to have an impaired sense of smell according to psychophysical olfactory testing. Olfactory dysfunction (OD), defined as the reduced or distorted ability to smell during sniffing (orthonasal olfaction) or eating (retronasal olfaction), is often reported in mild or even asymptomatic cases; in a study from Italy, 64\% of 202 mildly symptomatic patients reported impaired olfaction [3].

They concluded that the possibility that OD could act as a marker for disease, particularly among individuals who are otherwise minimally symptomatic or asymptomatic, prompted organizations, such as the American Academy of Otolaryngology-Head and Neck Surgery 4 and ENT UK,5 to recommend inclusion of sudden-onset loss of smell and/or taste as part of the diagnostic criteria for COVID-19 disease, as has now been done by the CDC. These organizations suggest that new-onset OD is sufficient to justify self-isolation and the use of personal protective equipment (PPE) by medical staff evaluating patients with this clinical problem.

Five patients in our study had only smell abnormality, and in fact that was the mode of presentation to our out patient clinic for which COVID test was done subsequently and they all were found to be positive. This also confirms olfactory abnormality especially a sudden olfactory loss should necessitate a diagnosis of COVID or have a high suspicion for positivity in the least [8]. 
Prasun Mishra performed a similar study to study the prevalence of Anosmia in confirmed COVID-19 patients, in Indian population and to ascertain its significance as a symptom of COVID 19. Study was done at a tertiary care COVID treating hospital. While eliciting detailed history from Covid-19 positive patients, all patients were asked about symptom of Anosmia. Same was asked from control group of subjects who were COVID-19 negative. The history of Anosmia was also elicited on discharge after the patients tested negative for COVID-19. 74 patients formed part of the study. 11 of 74 (14.8\%) patients had Anosmia. On using the chi square test for significance the difference was significant $(p<.01)$, suggesting Anosmia to be a significant clinical feature in COVID-19 patients. On comparing with world literature it was observed that the prevalence of Anosmia is higher in European population as compared to Indian Also the symptom of Anosmia improved when the patient recovered from the disease. Prevalence of new onset Anosmia in Indian population with COVID-19 is $14.8 \%$. Symptom of Anosmia in present times should be considered as a important clinical feature and should raise a suspicion of COVID-19. It was concluded that the prevalence of Anosmia in Indian population is much lesser than that reported in European population [9].

Our prevalence rate was 36 in hundred patients assessed by us. This was clinically significant as assessed by the Chi square analysis. This proves that olfactory anomalies are a significant symptom in the clinical scenario of COVID.

In May 2020 Anosmia was recognised as a symptom of covid-195 in light of accumulating evidence, including a meta-analysis which showed a loss of smell in 55\% (95\% confidence interval 38\% to $70 \%$ ) of patients with covid-19 [6]. A large online questionnaire based survey found that, in covid-19, loss of smell is usually severe and sudden in onset, but transient in most patients, although $10.6 \%$ (95\% CI, 5.6 to 17.8 ) of patients showed no improvement at one month. 7 It is advisable, therefore, to test for covid-19 infectioneither with reverse transcription polymerase chain reaction (RTPCR) (if presenting within 7 - 10 days of symptom onset) or serology (if presenting after 10 days)-in any patients with sudden onset loss of smell for whom there is no obvious alternative explanation (e.g. obstructing nasal mass, severe allergic rhinitis) and where the SARS-CoV-2 virus is active locally. Most patients with loss of smell and covid-19 infection will report other symptoms, although $16 \%$ of patients may have Anosmia as an isolated symptom.
Our study of 100 patients had 36 percent with loss of smell and reduced smell, this was a significant finding as assessed by the Chi Square analysis [10].

\section{Conclusion}

COVID is a learning experience and many new research and findings are coming almost everyday. In our study we have seen Olfactory anomalies such as Anosmia and hyposmia in around 36 percent of our patients. Thus and it is a major symptom of COVID. Most of the time (over 98 percent) Olfactory anomalies in COVID recover completely whatever treatment is given and this is a finding across different studies including the present one.

Females have a higher incidence of olfactory anomalies in COVID and this was significant according to the Chi Square analysis (26 vs 10). Further analysis on a larger scale is required though. Patients with olfactory complaints normally have good prognosis in COVID but further study is required since this may be an incidental association and our study did not find any significant association ( $p=0.924213$ ). Further analysis of route of infection especially the olfactory area may give important insights into the management of this pandemic. This may possibly be an area of interest in the development of an intra nasal vaccine against COVID.

\section{Bibliography}

1. Moran DT., et al. "The ultra- structure of the human olfactory musoca”. New York: Springer-Verlag (1991): 3-28.

2. Scherer PW., et al. "The biophysics of nasal airflow". Otolaryngologic Clinics of North America 22 (1989): 265-278.

3. Leopold DA. "The relationship between nasal anatomy and function". Laryngoscope 98 (1988): 1232-1238.

4. Schneider RA and Wolf S. "Relation to olfactory acuity to nasal membrane function". Journal of Applied Physiology 15 (1960): 914-920.

5. Doty RL and Mishura A. "Olfaction and its alteration by nasal obstruction, rhinitis, and rhinosinusitis". Laryngoscope 11 (2001): 409-423.

6. Gane SBC., et al. "Isolated Sudden onset anosmia in COVID-19 infection. A novel syndrome?" Rhinology (2020): 58. 
7. Han AY., et al. "Anosmia in COVID-19: Mechanisms and Significance" (2020).

8. Katherine Lisa Whitcroft and Thomas Hummel. "Olfactory Dysfunction in COVID-19; Diagnosis and Management". JAMA: The Journal of the American Medical Association 323.24 (2020): $2512-2514$.

9. Prasun Mishra., et al. "Prevalence of New Onset Anosmia in COVID-19 Patients: Is The Trend Different Between European and Indian Population?" The Indian Journal of Otolaryngology and Head and Neck Surgery 72.4 (2020): 484-487.

10. Abigail Walker., et al. "Anosmia and loss of smell in the era of covid-19". British Medical Journal (2020): 370.

Volume 3 Issue 9 September 2021

(C) All rights are reserved by Minutha $\mathrm{R}$. 